\title{
Motor control times and strategies in left- and right-handed participants: Behavioral evidence for the hemispheric distribution of motor planning
}

\author{
Neda Sadeghi, ${ }^{1,2}$, Mohammad Ali Nazari², Ali Shahbazi ${ }^{1,2}$, Mohammad Taghi Joghataei ${ }^{1,2 * \mathbb{D}}$ \\ Received: 26 May 2020 \\ Published: 24 Mar 2021
}

Abstract

Background: There is conflicting evidence in favor of the hemispheric distribution of motor planning. Some studies supported the left-hemisphere-dominance hypothesis for motor planning and claimed that the left-hemisphere has a crucial function in motor control even in left-handers. The present study aimed to compare the right- and left-handed participants on motor planning ability and to investigate the performance of their dominant hands in a specific action selection task. Also, the effect of task complexity was assessed.

Methods: Twenty right-handers and 20 left-handers performed an action selection task. The participants had to grasp a hexagonal knob with their dominant hand and consequently rotated it $60^{\circ}$ or $180^{\circ}$ clockwise or counterclockwise. Depending on our objects, we used mixed factorial ANOVA and the groups were examined in terms of the planning time, grasping time, releasing time and planning pattern for initial grip selection. The SPSS 19 was used for analyzing the data and $p \leq 0.05$ was considered as the significant level.

Results: No significant differences were observed between the two groups. The movement-related measures revealed a main effect of rotation $(p<0.001)$. However, a significant interaction between direction $\times$ planning pattern $\times$ group $(p<0.001)$ indicated a preferential bias for rotatory movements in the medial direction which is consistent with the "medial over lateral advantage".

Conclusion: Both left- and right-handed participants had a similar motor planning ability while performing a planning task with their dominant hands. Because our study was behavioral, it only provided a test of the left-hemisphere hypothesis of motor planning.

Keywords: Motor Control, Hemispheric Specialization, Handedness

Conflicts of Interest: None declared

Funding: Cognitive Sciences and Technologies Council

*This work has been published under CC BY-NC-SA 1.0 license.

Copyright $₫$ Iran University of Medical Sciences

Cite this article as: Sadeghi N, Nazari MA, Shahbazi A, Joghataei MT. Motor control times and strategies in left- and right-handed participants: Behavioral evidence for the hemispheric distribution of motor planning. Med J Islam Repub Iran. 2021 (24 Mar);35:39. https://doi.org/10.47176/mjiri.35.39

\section{Introduction}

The current knowledge about motor planning is that motor actions should be planned before they are executed in order to meet the purpose $(1,2)$. By definition, motor

\section{Corresponding author: Dr Mohammad Taghi Joghataei, joghataei@iums.ac.ir}

1. Cellular and Molecular Research Center, Iran University of Medical Sciences, Tehran, Iran

2. Department of Neuroscience, Faculty of Advanced Technologies in Medicine, Iran University of Medical Sciences, Tehran, Iran planning is the development of an action strategy with consideratio $\mathrm{n}$ for the future demands of the task, and by going beyond the immediately available motor-perceptual

\section{$\uparrow$ What is "already known” in this topic:}

Motor planning is the development of an action strategy with consideration for the future demands of the task and environment. There is conflicting evidence in favor of the hemispheric distribution of motor planning, with some supporting the left-hemisphere-dominance hypothesis for motor planning and some disclaiming it.

\section{$\rightarrow$ What this article adds:}

Previous studies do not provide strong evidence about the hemispheric distribution of motor planning. There are a few studies that focused on motor planning in left-handers. We performed a behavioral study in which the motor planning process was examined in left- and right-handed participants to test the left-hemisphere dominance hypothesis. 
information $(3,4)$. Action selection is an important step of motor planning which requires the selection of the right motor programs $(5,6)$. For example, people choose different grips when they want to write with a pen compared to when they want to pass it on to someone else (7). There has been a growing interest in motor planning and its hemispheric distribution (8-10). Anatomical evidence shows that pyramidal cells in the primary motor cortex mostly project to contralateral alpha-motoneurons in the brain stem and spinal cord, so it's reasonable for each hemisphere to control the motor activity of the contralateral limb. However, our body moves as a whole, with limbs working together in unified patterns. Therefore, there must be planning centers to program the motor activity of both body-sides as a unit. However, arguing about hemispheric distribution of these planning centers remains difficult. Motor planning processes have an essential role in behavioral performance of neurologic patients $(11,12)$ and athletes (13-15). So, understanding the nature of motor planning and its underlying mechanisms is important to target them effectively in therapeutic and sport contexts.

Some studies suggested a left-hemisphere specialization for motor planning (16-19). For example, Schluter et al. (18) investigated cerebral dominance for action selection processes using Positron-Emission Tomography (PET). The activated networks were in the left premotor, left prefrontal and left intraparietal areas. The authors suggested that, in addition to speech, the left side of the brain is also prepotent for action. However, the evidence has relied only on right-handers, which is an important limitation. The left-hemisphere is the dominant brain in right-handers and the results may be due to experience and practice with right hand instead of a generic left-hemisphere specialization for motor planning. There are a few studies on the field of motor control in left-handers. Janssen et al. (17, 20) investigated the motor planning process in right- and left-handed participants using a bimanual grasping task. The participants were trained to grasp CD cases then put them in CD boxes with a specific orientation. The authors found that the end-state comfort effect was only apparent for the right hand in both groups. The results from a study on split-brain patients (1 right-hander and 1 left-hander) showed that the right hand performance was superior to the left in both groups when performing a tool-use task (21). Also an fMRI study on left-handed participants showed that left-lateralized motor areas were activated in both right- and left-handed participants during a pantomime task (22). Although these studies have supported the left-hemisphere dominance for motor planning even in left-handers, the conclusions were based on either the data of only two participants (21) or using motor tasks similar to the daily actions, like putting $\mathrm{CD}$ cases on $\mathrm{CD}$ boxes $(17,20)$ and pantomiming tool use $(22)$, which are compatible for right-handers, but left-handers also adapted to do these actions with their right hand because of living in a 'right-handed world' (23). In contrast, some studies demonstrated right hemisphere dominance for manual motor skills in right- and left-handers. For example, Kilshaw and Annett (24) investigated hand skills in healthy right- and left-handed participants. Left-handed participants were faster than right-handers in several tests by the preferred hand. Also Tankle and Heilman (25) showed that when performing a mirror writing task, left-handed participants made fewer mistakes than did right-handed participants using the preferred hand. In addition, the left hand performance was better in both groups. Hughes et al. (26) found no difference in motor planning between leftand right-handers and initial grasp postures were similar for both the preferred and non-preferred arm, but motor execution was influenced by hand used. The left hand had shorter transport times in both groups. These mixed results from studies on left-handed populations, make it difficult to draw a concrete conclusion about hemispheric distribution of motor planning.

Studies on patients with unilateral brain damage have another view to investigate hemispheric distribution of motor planning. Some evidence supports left hemisphere dominance for motor planning, claiming that patients with left brain damage suffer from more severe motor planning deficits than do patients with right brain damage. For example, Craje et al. (27) compared 22 patients with right and left Hemiparetic Cerebral Palsy (HCP) during a sequential grasping task. The experimenter instructed patients to grasp a rod in a rod-and-frame illusion with a proper grip. The results showed that the right HCP group (with lesions in the left hemisphere) had remarkable planning problems compared to the other group. Steenbergen et al. (4) also suggested that motor planning deficits were more severe in people whose right side of the body was affected. Nonetheless, Kirkpatrick et al. (28) found no differences between children with right and left HCP when performing an action planning task with the unaffected arm. Dean et al. (29) also suggested that no differences were observed between stroke patients with right and left hemisphere lesions. Williams et al. (30) showed that both children with the right and left spastic hemiplegia had similar response time and accuracy rate on the hand rotation task. These latter studies are inconsistent with the left-hemisphere-dominance hypothesis for motor planning.

Therefore, previous evidence in favor of lefthemisphere dominance in motor planning is still challenging. However, Baraldi et al. (31) proposed a widespread system across the brain for planning the objective motor programs for each limb. They suggested that bilateral regions in the motor cortex have an important role in anticipating planning of actions. If the left-hemispheredominance hypothesis is true, the left side of the brain has a generic specialization in motor planning, regardless of handedness. With such an assumption one can expect the dominant-left-hemisphere to overcome the dominantright-hemisphere in a planning task. To address this issue, we investigated the performance of the dominant hands (corresponding to dominant brains) in right- and lefthanded participants while performing a motor planning task. Generally, motor planning is assessed based on the "end-state comfort effect", i.e. that people often show a tendency to end tasks with a comfortable posture, even if they have to start it with an uncomfortable hand posture 
$(32,33)$. In the present study we used a specific action selection task in which participants needed to decide how to rotate their dominant hands and select the proper grip for grasping and rotating a hexagonal knob in different rotational angles and directions. Different levels of motor process were studied by this task, including the preparation phase (before movement onset) and the execution phase (after voluntary movement). The primary goal of the study was to compare the performance of the dominant hands in right- and left-handed participants during a specific action selection task. We investigated whether there is a difference between right- and left-handed participants in planning times, grasping times, releasing times and planning patterns for initial grip selections. In addition, it is suggested that more complex actions (i.e. rotating a knob over a larger angle) would require stronger planning processes $(34,35)$. Therefore, it was also hypothesized that complex planning conditions would manifest in slower movement-related times and better planning pattern for initial grips.

\section{Methods}

\section{Participants}

The experiment involved 40 adolescents (20 lefthanders and 20 right-handers) in the age range of 11 to 19 years, with a mean age of $14.72 \pm 2.1$ years. There was no difference in terms of age or gender between groups. Handedness was tested by the four-item version of the Edinburgh Handedness Inventory (scores $\geq 61$ indicated right-handedness and $\leq-61$ indicated left-handedness) (36), and the mean score was $98.75 \pm 3.8$ for the righthanders and $-90 \pm 14.3$ for the left-handers. All the participants had normal or corrected vision and were neurologically healthy, with no sign of developmental coordination disorder (DCD) or any known neuromuscular disease. Data were obtained from their medical profiles or parent interviews. All participants included in our study signed an informed assent. They were blinded to the goal of the reseach and had received participation rewards in advance. All procedures involving human participants were approved by the research committee of Iran University of Medical Science (IR.IUMS.FMD.REC1396.9321959005) in accordance with the ethical standards of the institutional and national research committee and with the 1964 Helsinki declaration. Informed consent was obtained from all participating authorities, and children provided verbal assent.

\section{Experiment setup}

Figure 1A presents the custom-made apparatus used in this experiment. This apparatus was similar to the setup used by Mutsaarts et al. (3) and was placed on a table in a quiet room. The participants were instructed to sit comfortably on a chair behind this table and adjust the seat height in order to perform the task in a suitable posture. The setup consisted of a custom-made portable wedge (width: $40 \mathrm{~cm}$ ) with a $45^{\circ}$ - slope relative to the horizontal surface. A plastic hexagonal knob (width: $11 \mathrm{~cm}$; depth: 6 $\mathrm{cm})$ was attached to the center of the wedge. The hexagonal knob could smoothly rotate around its vertical axis. A touch sensor which recorded the maximum touch surface was installed on each of the six sides of the knob to detect initial grip patterns. The participants were instructed to grasp the hexagonal knob with their thumb and fingers placed on opposite sides of the knob. Therefore only two opposite sides of the hexagon were touched and the location of the fingers in front of the thumb (the surface with maximum touch) was detected by the sensor. So there were six grip patterns (Grip 1, Grip 2, Grip 3, Grip 4, Grip

A)

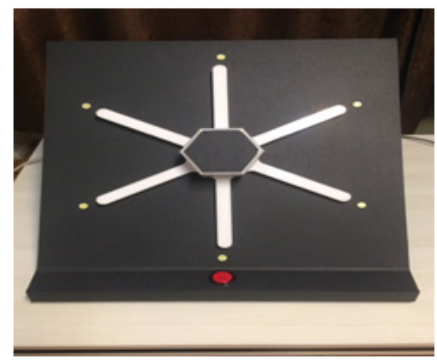

B)
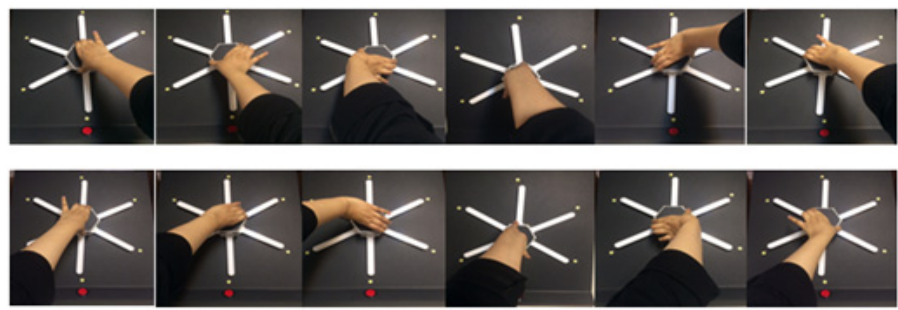

C)

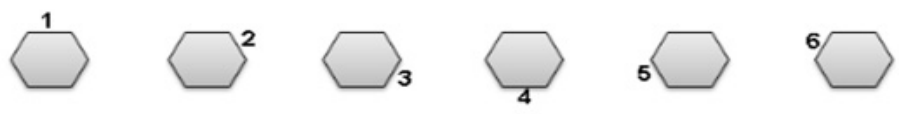

Fig. 1. A) the custom-made apparatus for motor planning assessment adapted from Mutsaart (2006). B) The six grip patterns with right hand (upper panel) and left hand (lower panel), separately. Grip 4 was biomechanically impossible for all the participants. C) Diagrams indicating 6 possible locations of four fingers in front of thumb detected by touch sensor (6 initial grips). 
5 and Grip 6). Grip 4 was biomechanically impossible for all the participants (Fig. 1B and C). An arrow (width: 0.5 $\mathrm{cm}$; length: $15 \mathrm{~cm}$ ) was inserted into each of the six sides of the knob. At $0.5 \mathrm{~cm}$ from the edge of the wedge, six LEDs were placed at $0^{\circ}, 60^{\circ}, 120^{\circ}, 180^{\circ}, 240^{\circ}$ and $300^{\circ}$. There was a response button at the bottom and front of the wedge to allow the participants to start the grasping movements. Both the response button and portable wedge were controlled by a PC running presentation software (motor planning system, TIWAN Technology Development Co.) to turn on the LEDs to record the planning time (i.e. the time in which the subject released the response button), grasping time (i.e. the time between releasing the response button and grasping the hexagon) and releasing time (i.e. the time between grasping and rotating the hexagon) and to detect the rotation angles and directions.

\section{Experiment procedure}

The experiment started with the dominant hand placed on the response button. By pressing the button, a number of LEDs turned on and the initial trial began. The LEDs instructed four different conditions in terms of direction and rotation angle, including $60^{\circ}, 180^{\circ}$ clockwise $(\mathrm{CW})$ or $60^{\circ}, 180^{\circ}$ counterclockwise (CCW). For example, if LEDs $1,6,5$ and 4 were lit, it meant that the hexagon was required to rotate $180^{\circ}$ in a counterclockwise direction (Fig. 2A). The participants had to release the response button after they had made their decision about which grip they wanted to choose. They then grasped the hexagon and rotated it in the pre-cued direction and rotation angle. The participants were free to choose from five possible grips. Each time a rotation was carried out, the hand returned on the response button to initiate the next trial. Generally, the experiment consisted of 120 trials ( 2 directions $\times 2$ rotation angles $\times 30$ repetitions), which were presented in a random order.

The participants were told to perform the task as fast as possible with their dominant hand. Before the experiment, the participants were asked to put their hand over the hexagon and find the most comfortable grip. They all reported grip 1 (the without rotation grip) as the most comfortable grip. Before starting the task, participants practiced a few trials to learn the task and make sure whether the experiment was realized correctly.

\section{Data analysis}

Analysis of the stored data focused on planning times, grasping times, releasing times and planning pattern for initial grip selections as our outcome measures. Planning time was the time between the LEDs switching on and the release of the response button, grasping time was the time between the release of the response button and grasping the hexagon and releasing time was the time between grasping the hexagon and releasing it after rotation (Fig. $2 \mathrm{~B})$. The responses that exceeded $\pm 3 \mathrm{SD}$ of the mean were excluded from the next steps of the analysis.

Initial grips were coded according to the location of four fingers in front of the thumb (Fig. 1C). For analyzing the motor planning process in initial grip selections, three planning patterns (no-planning pattern, effective-planning pattern and wrong-planning pattern) were defined. The without rotation grip (grip 1) was defined as the noplanning pattern. This pattern occurs due to the habitual system domination which results in using simple ways
A)

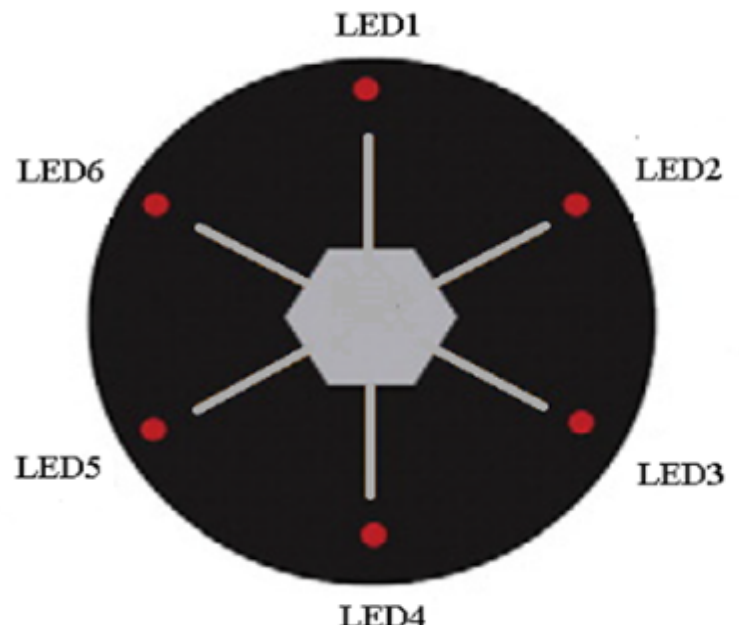

$\begin{array}{ll}\text { LEDs } & \text { Conditions } \\ 1+2= & 1\left(60^{\circ} \mathrm{CW}\right) \\ 1+2+3+4= & 2\left(180^{\circ} \mathrm{CW}\right) \\ 1+6= & 3\left(60^{\circ} \mathrm{CCW}\right) \\ 1+6+5+4= & 4\left(180^{\circ} \mathrm{CCW}\right)\end{array}$

$1+6+5+4=4\left(180^{\circ} \mathrm{CCW}\right)$

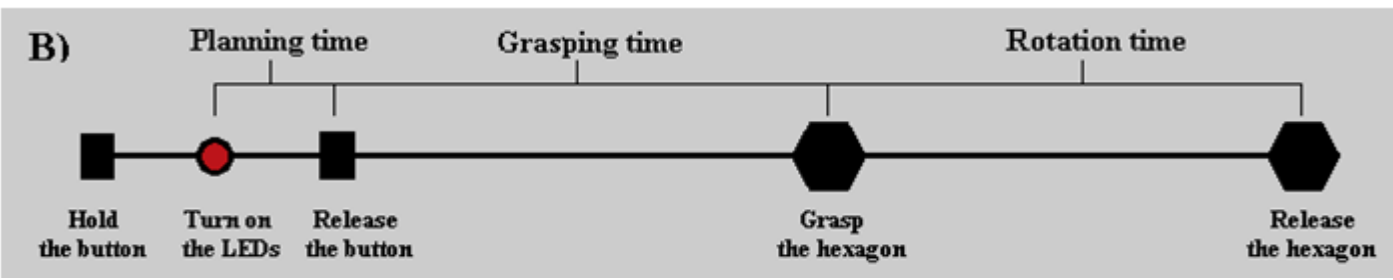

Fig. 2. A) Schematic design of the experimental setup and LED-based instructions. B) A trial of the task: holding the button, turning on the LEDs, releasing the button, grasping the hexagon and finally releasing the hexagon. 
without considering future demands of actions. The noplanning pattern only provides the end-state comfort satisfaction for 60 degree rotations. In order to perform the task in comfortable positions, the subjects had to initially rotate their hands in the opposite direction of the instructed rotatory movements. So, grip 5 and grip 6 for rotatory movements in $\mathrm{CW}$ directions and grip 2 and grip 3 for rotatory movements in $\mathrm{CCW}$ directions were defined as effective-planning patterns. The effective-planning pattern was an indicator of using the goal-directed system that anticipates the future consequences of upcoming actions. If the subjects initially rotated their hands in the same direction as the instructed rotatory movements, they could not complete the task or their hands ended in an uncomfortable and awkward position. For rotatory movements in CW directions, grip 2 and grip 3 and for rotatory movements in CCW directions, grip 5 and grip 6 were defined as wrong planning patterns.

\section{Statistical analysis}

Normality of data was confirmed by the KolmogorovSmirnov normality test (37). The statistical analysis was separately applied for planning times, grasping times, and releasing times. A mixed factorial ANOVA ( 2 directions $\times$ 2 rotation angles $\times 2$ groups) with Direction (counterclockwise vs. clockwise) and Rotation angle $\left(60^{\circ}\right.$ vs. $180^{\circ}$ ) as within-subject variables and Group (right- vs. left-handed subjects) as between-subjects factor was used. The planning pattern for initial grip selections was analyzed using a mixed factorial ANOVA ( 2 directions $\times 2$ rotation angles $\times 3$ planning patterns $\times 2$ groups) with Direction (counterclockwise vs. clockwise), Rotation angle $\left(60^{\circ}\right.$ vs. $\left.180^{\circ}\right)$ and Planning pattern (no-planning pattern, effective-planning pattern and wrong-planning pattern) as within-subject variables and Group (right- vs. left- handed subjects) as between-subjects factor. $\mathrm{P} \leq 0.05$ was considered as statistically significant (38).

\section{Results}

Demographic information was collected from all participants and statistical values are presented in Table 1. The analysis of planning times showed a significant main effect of rotation $\left[\mathrm{p}<0.001, \mathrm{n}^{2}=0.37\right]$, reflected the slower planning times for rotation angles $180^{\circ}$ compared to $60^{\circ}$ (Fig. 3A). In other words, increasing the rotation angles led to an increase in the planning time. There was no significant effect of direction $\left[p=0.410, \eta^{2}=0.01\right]$. No effect of group was observed $\left[p=0.480, \eta^{2}=0.01\right]$.

The analysis of grasping times revealed a significant main effect of rotation $\left[\mathrm{p}<0.001, \mathrm{\eta}^{2}=0.68\right]$, indicated slower grasping times for rotation angles $180^{\circ}$ compared to $60^{\circ}$ (Fig. 3B). There was no significant effect of direction $\left[p=0.130, n^{2}=0.05\right]$. Meanwhile, there was a significant interaction between direction $\times$ group $[\mathrm{p}=0.040$, $\left.\mathrm{n}^{2}=0.1\right]$, suggesting slower grasping times for $\mathrm{CW}$ directions in right-handed participants compared to the other group (Fig. 4). No effect of group was observed $[p=0.25$, $\left.\eta^{2}=0.03\right]$

The analysis of releasing times revealed a significant main effect of rotation $\left[\mathrm{p}<0.001, \mathrm{n}^{2}=0.86\right]$, reflected slower rotation times for $180^{\circ}$ compared to $60^{\circ}$ (Fig. 3C). There was no significant effect of direction $[\mathrm{p}=0.68$, $\left.\mathrm{n}^{2}=.005\right]$. Main effect of the group was not significant $\left[\mathrm{P}=0.15, \mathrm{n}^{2}=0.05\right]$.

The analysis of planning pattern for initial grip selection revealed a significant main effect of planning pattern $[\mathrm{P}<$ $\left..001, \mathrm{n}^{2}=.89\right]$, indicating the most planning pattern used by subjects was effective-planning pattern. There was no significant interaction between planning pattern $\times$ group $\left[p=0.650, \eta^{2}=0.01\right]$, reflecting that both groups used the same planning pattern. There was no significant interac-

Table 1. Mean \pm standard deviation with $\mathrm{t}$-test $(\mathrm{t})$ of age and chi-square test $\left(\dot{\mathrm{X}}^{2}\right)$ of gender for groups

\begin{tabular}{lccc}
\hline & Right-handers & Left-handers & $\mathrm{p}$ \\
\hline Number & 20 & 20 & \\
Age (years) & $14.30 \pm 1.59$ & $15.15 \pm 2.62$ & 0.220 \\
Gender & 9 male, 11 female & 11 male, 9 female & 0.520 \\
\hline
\end{tabular}

A)

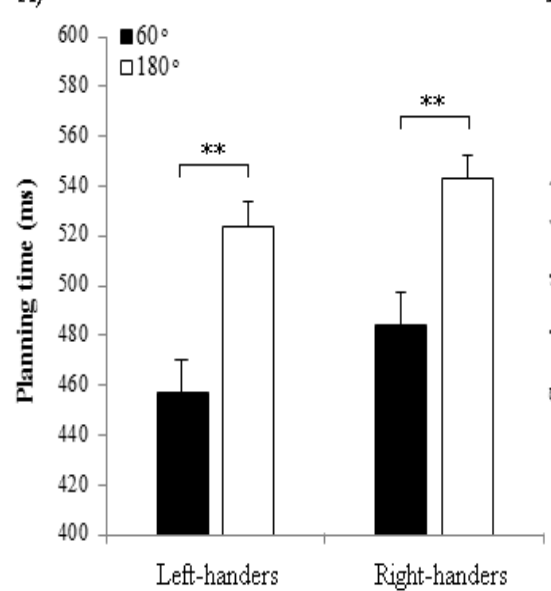

B)

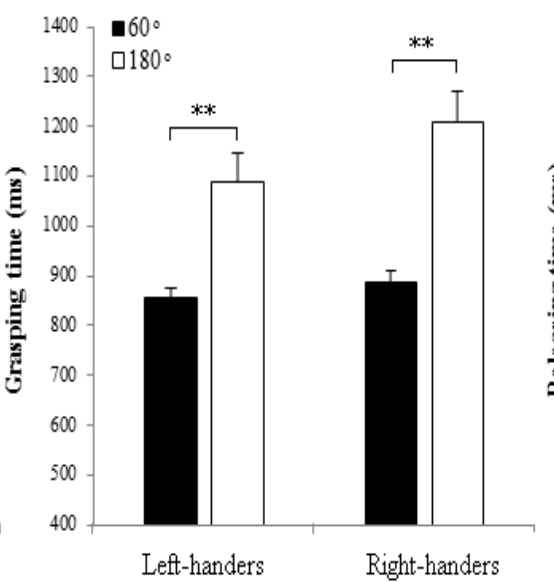

C)

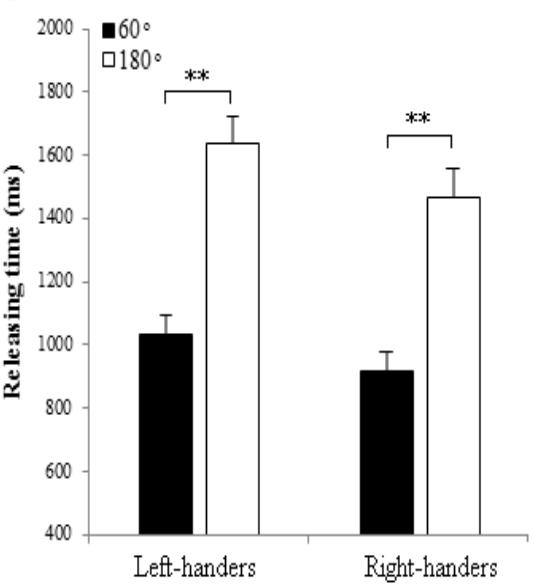

Fig. 3. Graphs representing planning times (A), grasping times (B), releasing times (C) for left- and right-handed participants. Error bars indicate $+1 \mathrm{SE}$. 


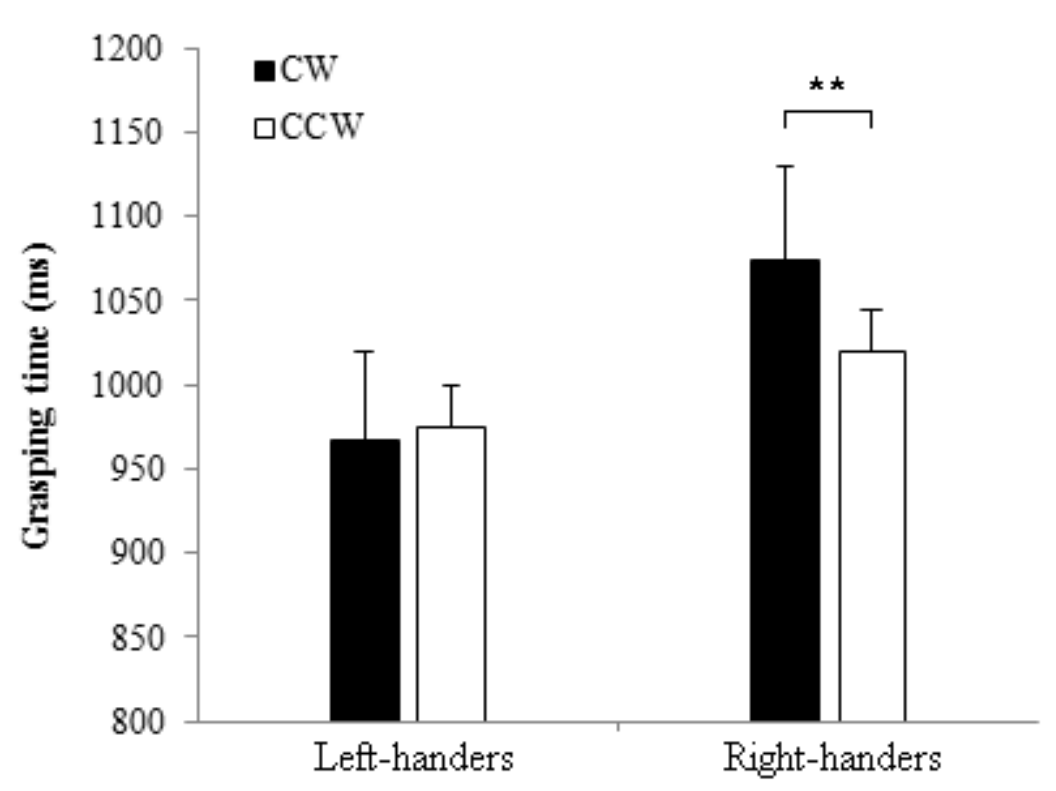

Fig. 4. Grasping times value for $\mathrm{CW}$ (black bars) and $\mathrm{CCW}$ (white bars) directions in left- and right-handed participants. Error bars indicate $+1 \mathrm{SE}$

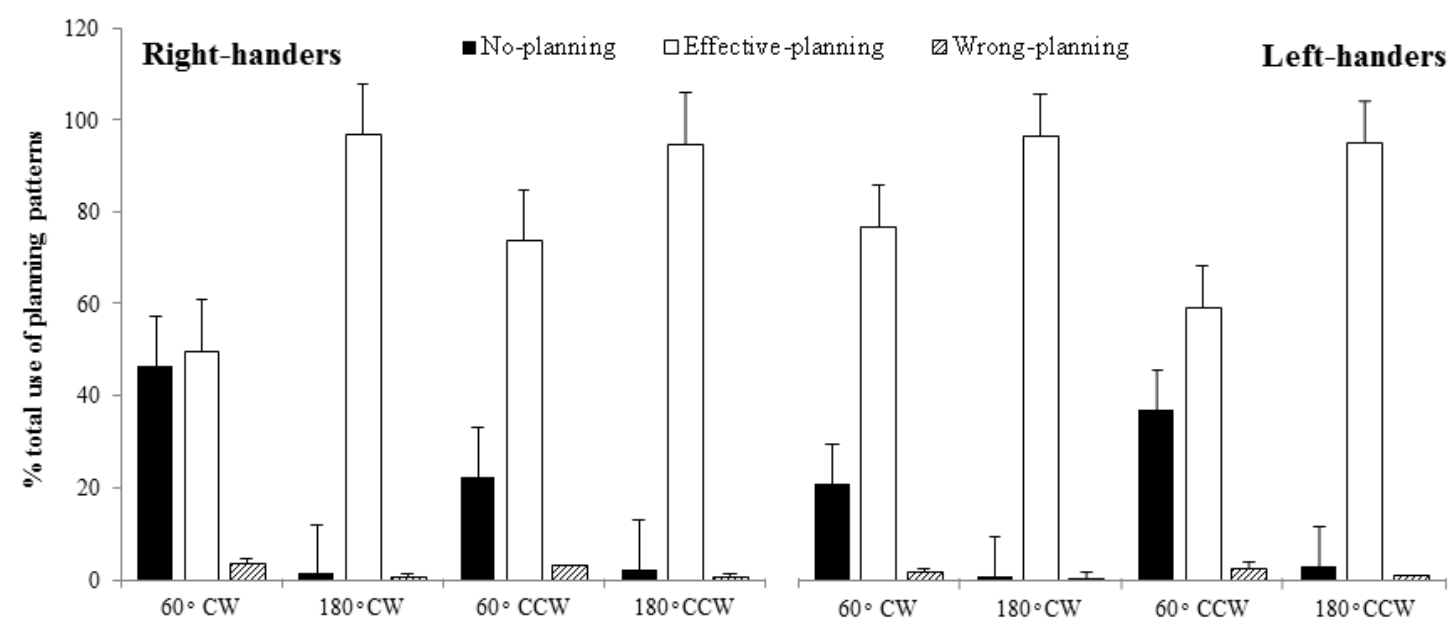

Fig. 5. Distribution of planning patterns used by right-handers (left) and left-handers (right) in four different conditions. Error bars indicate $+1 \mathrm{SE}$

tion between planning pattern $\times$ direction $[\mathrm{p}=0.830$, $\left.\mathrm{n}^{2}=0.005\right]$. There was a significant interaction between planning pattern $\times$ rotation $\left[\mathrm{p}<0.001, \mathrm{n}^{2}=0.54\right]$, indicating effective-planning patterns more often used for $180^{\circ}$ rotations, whereas subjects preferred the no-planning pattern for $60^{\circ}$ rotations. There was a significant interaction between planning pattern $\times$ direction $\times$ group $[p<0.001$, $\left.\eta^{2}=0.29\right]$, reflected that right-handed participants had a preference of choosing more effective-planning patterns in $\mathrm{CCW}$ directions and more no-planning patterns in $\mathrm{CW}$ directions, whereas left handed participants showed an opposite preference. These results were depicted in Figure 5.

\section{Discussion}

In the present study we investigated motor planning and execution processes during an action selection task, and sought to determine whether there is a difference between right- and left-handed participants performing the task with their dominant hands. The findings revealed similar motor planning and execution abilities in both groups. Given that a brain process is not an "all or nothing" phenomenon, and even people with neurological disorders use these processes one way or another $(35,39)$, this research did not intend to find a motor planning deficit in one group. Based on the left-hemisphere-dominance hypothesis for motor planning, it was expected that dominant right hand (in right-handers) have an advantage to dominant left hand (in left-handers) during the task performance. The results showed no difference between right- and lefthanded participants in an action selection task using their dominant hands (corresponding to dominant brains). Thus the present study did not confirm the left-hemispheredominance hypothesis for motor planning.

There are contradictory results in favor of lefthemisphere-dominance hypothesis for motor planning. Some studies supported this hypothesis by studying motor planning only in right-handed participants (9, 16-19). A 
few studies on motor control in left-handers showed mixed results. Some of them found a left-hemispheredominance for motor planning in both right- and lefthanded participants (20-22). However, some evidence was not in line with left-hemisphere-dominance hypothesis for motor planning and revealed a superior performance for the left hand even in right-handers (24-26). For example, Janssen et al. $(17,20)$ showed a prominent end-state comfort effect for the right hand in both right- and left-handed participants using a CD placing paradigm. However, Hughes et al. (26) using a different task, found no difference in initial grasping postures for both the dominant and non-dominant hand, regardless of handedness. In fact these conclusions might be specific to different experimental task and paradigm used in their studies. According to Serrien et al. (10) lateralization of motor function is an adaptable process in which both hemispheres have a flexible involvement. Also motor planning is a task-dependent process that is related to the task context and the precision of movement $(40,41)$. Furthermore, some studies on patients with unilateral brain damage claimed that patients with lesions in the left hemisphere disproportionately suffer from motor planning deficits $(4,27)$. Whereas there were studies that did not confirm that claim (28-30). So it is difficult to draw a reliable conclusion to support the left-hemisphere-dominance hypothesis and further research is needed.

An increase in rotation angle was found to be associated with increased movement-related times (planning times, grasping times and releasing times). This finding might be explained by noting that an increased rotation angle and task complexity require more processing and execution times. Marteniuk et al. (41) showed that the participants acted more slowly in precision tasks (e.g. fitting a small object into a small hole). At the neural level, Van Elk et al. (35) showed that an increased rotation angle was associated with higher amplitude of P2 which is the neural component of action selection.

The analysis of planning patterns for initial grip selection showed that all the participants used effectiveplanning patterns more than other patterns (no-planning and wrong-planning pattern). The finding that more effective-planning patterns were observed in rotation angle $180^{\circ}$ compared to $60^{\circ}$ can be explained by the competition between the habitual and the goal-directed system. The habitual system always uses simple ways without considering further action sequences while the goal-directed system anticipates both current and future task demands before the execution of action. Accordingly, in simple planning conditions (i.e. $60^{\circ}$ rotations), the habitual and the goal directed systems can easily perform the task and achieve end-state comfort satisfaction; however, in complex planning conditions (i.e. $180^{\circ}$ rotations), end-state comfort satisfaction can be obtained only by the goaldirected system $(1,32,40)$. In fact, subjects had to use the goal-directed system for the $180^{\circ}$ rotations and immediately turn their hands in the opposite directions of the instructed rotatory movements. Otherwise, they could not complete the task or their hands ended in an uncomfortable and awkward position. In line with this finding,
Stöckel et al. (42) also suggested that the increased complexity and precision of a task leads to an increased sensitivity toward stronger planning process. However, a significant interaction between direction $\times$ planning pattern $\times$ group indicated a preferential bias for rotatory movements in the medial direction which is consistent with the "medial over lateral advantage". The right-handed participants showed a preference of choosing more effective-planning patterns in counterclockwise directions and more noplanning patterns in clockwise directions, whereas left handed participants had an opposite preference (Fig. 5). Literally, for the right-handed participants, a CW rotation is a lateral one, and a CCW rotation is a medial one. However, for the left-handed participants the opposite is true a $\mathrm{CW}$ rotation is a medial one and a $\mathrm{CCW}$ rotation is a lateral one.

\section{Conclusion}

There are a few studies that focused on motor planning in left-handers. In the present study we examined motor planning in left- and right-handed participants to test the left-hemisphere dominance hypothesis. In general, we found no difference between right- and left-handed participants using their dominant hands (corresponding to dominant brains) in an action selection task. Because our study was behavioral, it only provided a test of the lefthemisphere hypothesis of motor planning. The existing evidence mandates further research on this topic using more precise techniques, like electrophysiological techniques (ERPs).

\section{Acknowledgments}

This work was supported by the Cognitive Sciences and Technologies Council. TIWAN Technology Development Co. constructed the apparatus. The brain and cognition clinic helped us with data collection.

Conflict of Interests

The authors declare that they have no competing interests.

\section{References}

1.Johnson-Frey S, McCarty M, Keen R. Reaching beyond spatial perception: Effects of intended future actions on visually guided prehension. Vis Cogn. 2004;11(2-3):371-99.

2. Mutsaarts M, Steenbergen B, Bekkering H. Anticipatory planning of movement sequences in hemiparetic cerebral palsy. Motor Control.2005;9(4):439.

3. Mutsaarts M, Steenbergen B, Bekkering H. Anticipatory planning deficits and task context effects in hemiparetic cerebral palsy. Exp Brain Res. 2006;172(2):151-62.

4. Steenbergen B, Meulenbroek RG, Rosenbaum DA. Constraints on grip selection in hemiparetic cerebral palsy: effects of lesional side, end-point accuracy, and context. Cogn Brain Res. 2004;19(2):145-59.

5. Andersen RA, Cui H. Intention, action planning, and decision making in parietal-frontal circuits. Neuron. 2009;63(5):568-83.

6. Wong A, Haith A, Krakauer J. Motor planning. Neuroscientist. 2014.

7. Daprati E, Sirigu A. How we interact with objects: learning from brain lesions. Trends Cog Sci. 2006;10(6):265-70.

8. Cohen RG, Rosenbaum DA. Where grasps are made reveals how grasps are planned: generation and recall of motor plans. Experimental Brain Res. 2004;157(4):486-95.

9. Sabaté M, González B, Rodríguez M. Brain lateralization of motor imagery: motor planning asymmetry as a cause of movement 
lateralization. Neuropsychologia. 2004;42(8):1041-9.

10. Serrien DJ, Ivry RB, Swinnen SP. Dynamics of hemispheric specialization and integration in the context of motor control. Nat Rev Neurosci. 2006;7(2):160.

11. Steenbergen B, Gordon AM. Activity limitation in hemiplegic cerebral palsy: evidence for disorders in motor planning. Dev Med Child Neurol. 2006;48(09):780-3.

12. Steenbergen B, Verrel J, Gordon AM. Motor planning in congenital hemiplegia. Disabil Rehabil. 2007;29(1):13-23.

13. Bianco V, Di Russo F, Perri RL, Berchicci M. Different proactive and reactive action control in fencers' and boxers' brain. Neuroscience. 2017;343:260-8.

14. Del Percio C, Rossini PM, Marzano N, Iacoboni M, Infarinato F, Aschieri $\mathrm{P}$, et al. Is there a "neural efficiency" in athletes? A highresolution EEG study. Neuroimage. 2008;42(4):1544-53.

15. Hung T-M, Spalding TW, Santa Maria DL, Hatfield BD. Assessment of reactive motor performance with event-related brain potentials: attention processes in elite table tennis players. J Sport Exerc Psychol 2004;26(2):317-37.

16. Haaland KY, Elsinger CL, Mayer AR, Durgerian S, Rao SM. Motor sequence complexity and performing hand produce differential patterns of hemispheric lateralization. J Cogn Neurosci. 2004;16(4):621-36.

17. Janssen L, Beuting M, Meulenbroek R, Steenbergen B. Combined effects of planning and execution constraints on bimanual task performance. Exp Brain Res. 2009;192(1):61-73.

18. Schluter N, Krams M, Rushworth M, Passingham R. Cerebral dominance for action in the human brain: the selection of actions. Neuropsychologia. 2001;39(2):105-13.

19. Schluter N, Rushworth M, Passingham R, Mills K. Temporary interference in human lateral premotor cortex suggests dominance for the selection of movements. A study using transcranial magnetic stimulation. Brain. 1998;121(5):785-99.

20. Janssen L, Meulenbroek RG, Steenbergen B. Behavioral evidence for left-hemisphere specialization of motor planning. Exp Brain Res 2011;209(1):65-72.

21. Frey SH, Funnell MG, Gerry VE, Gazzaniga MS. A dissociation between the representation of tool-use skills and hand dominance: insights from left-and right-handed callosotomy patients. J. Cogn. Neurosci. 2005;17(2):262-72.

22. Frey SH. Tool use, communicative gesture and cerebral asymmetries in the modern human brain. Philos Trans R Soc Lond., B Biol Sci. 2008;363(1499):1951-7.

23. Gonzalez CL, Whitwell R, Morrissey B, Ganel T, Goodale MA. Left handedness does not extend to visually guided precision grasping. Exp Brain Res. 2007;182(2):275-9.

24. Kilshaw D, Annett M. Right-and left-hand skill I: Effects of age, sex and hand preference showing superior skill in left-handers. Br J Psychol. 1983;74(2):253-68.

25. Tankle RS, Heilman KM. Mirror writing in right-handers and in lefthanders. Brain Lang. 1983;19(1):115-23.

26. Hughes CM, Reißig P, Seegelke C. Motor planning and execution in left-and right-handed individuals during a bimanual grasping and placing task. Acta Psychol . 2011;138(1):111-8.

27. Crajé C, van der Kamp J, Steenbergen B. Visual information for action planning in left and right congenital hemiparesis. Brain Res. 2009; 1261:54-64.

28. Kirkpatrick E, Pearse J, Eyre J, Basu A. Motor planning ability is not related to lesion side or functional manual ability in children with hemiplegic cerebral palsy. Exp Brain Res. 2013;231(2):239-47.

29. Dean PJA, Seiss E, Sterr A. Motor planning in chronic upper-limb hemiparesis: evidence from movement-related potentials. PLoS One. 2012;7(10):e44558.

30. Williams J, Anderson V, Reddihough DS, Reid SM, Vijayakumar N, Wilson PH. A comparison of motor imagery performance in children with spastic hemiplegia and developmental coordination disorder. J Clin Exp Neuropsychol. 2011;33(3):273-82.

31. Baraldi P, Porro CA, Serafini M, Pagnoni G, Murari C, Corazza R, et al. Bilateral representation of sequential finger movements in human cortical areas. Neurosci Lett. 1999;269(2):95-8.

32. Rosenbaum DA, Marchak F, Barnes HJ, Vaughan J, Slotta JD, Jorgensen MJ. Constraints for action selection: Overhand versus underhand grips. 1990.

33. Rosenbaum DA, Meulenbroek RJ, Vaughan J, Jansen C. Posturebased motion planning: applications to grasping. Psychol Rev.
2001;108(4):709.

34. Smid HG, Jakob A, Heinze HJ. An event-related brain potential study of visual selective attention to conjunctions of color and shape. Psychophysiology. 1999;36(2):264-79.

35. Van Elk M, Crajé C, Beeren M, Steenbergen B, Van Schie H, Bekkering $\mathrm{H}$. Neural evidence for impaired action selection in right hemiparetic cerebral palsy. Brain Res. 2010;1349:56-67.

36. Veale JF. Edinburgh handedness inventory-short form: a revised version based on confirmatory factor analysis. Laterality. 2014;19(2):164-77.

37. Mehdizadeh M, Martinez-Martin P, Habibi SA. The Association of Balance, Fear of Falling, and Daily Activities With Drug Phases and Severity of Disease in Patients With Parkinson. Basic Clin Neurosci. 2019;10:4.

38. Mehdizadeh M, Martinez-Martin P, Habibi SA.Fereshtehnejad SM, Abasi A, Niazi Khatoon J, Saneii SH, Taghizadeh G. Reliability and validity of fall efficacy Scale-International in people with Parkinson's disease during on-and off-drug phases. Parkinsons Dis. 2019; doi.org/10.1155/2019/6505232.

39. Craje C, Aarts P, Nijhuis-van der Sanden M, Steenbergen B. Action planning in typically and atypically developing children (unilateral cerebral palsy). Res Dev Disabil. 2010;31(5):1039-46.

40. Claxton LJ, Keen R, McCarty ME. Evidence of motor planning in infant reaching behavior. Psychol Sci. 2003;14(4):354-6.

41. Marteniuk R, MacKenzie C, Jeannerod M, Athenes S, Dugas C. Constraints on human arm movement trajectories. Can J Psychol. 1987;41(3):365.

42. Stöckel T, Hughes CM, Schack T. Representation of grasp postures and anticipatory motor planning in children. Psychol Res 2012;76(6):768-76 